\title{
Nilotinib for the treatment of chronic myeloid leukemia:An evidence-based review
}

\author{
Elias Jabbour \\ Jorge Cortes \\ Hagop Kantarjian \\ Department of Leukemia, \\ The University of Texas M.D. \\ Anderson Cancer Center, \\ Houston, TX, USA
}

\author{
This article was published in the following Dove Press journal: \\ Core Evidence \\ 3 October 2009 \\ Number of times this article has been viewed
}

Introduction: Chronic myelogenous leukemia (CML) is a progressive and often fatal hematopoietic neoplasm. The Bcr-Abl tyrosine kinase inhibitor imatinib mesylate represented a major therapeutic advance over conventional CML therapy, with more than $90 \%$ of patients obtaining complete hematologic response, and $70 \%-80 \%$ of patients achieving a complete cytogenetic response. Despite the high efficacy of imatinib, a minority of patients in chronic phase CML and more patients in advanced phases are resistant to imatinib, or develop resistance during treatment. This is attributed, in $40 \%$ to $50 \%$ of cases, to the development of mutations in the Bcr-Abl tyrosine kinase domain that impair imatinib binding. Attempts to circumvent resistance led to the discovery of nilotinib (Tasigna), a novel, potent and selective oral Bcr-Abl kinase inhibitor.

Aims: To review the evidence for the use of nilotinib in the management of CML.

Evidence review: Preclinical and clinical investigations demonstrate that nilotinib effectively overcomes imatinib resistance, and has further improved the treatment of CML.

Place in therapy: Nilotinib is currently indicated for patients with CML in chronic and accelerated phases following imatinib failure. Randomized studies are ongoing to assess the efficacy of nilotinib in patients with newly diagnosed CML.

Keywords: CML, tyrosine kinase inhibitors, nilotinib, imatinib-resistance, imatinibintolerance.

\section{Core evidence place in therapy summary for nilotinib in CML}

\begin{tabular}{|c|c|c|}
\hline Outcome measure & Evidence & Implications \\
\hline \multicolumn{3}{|c|}{ Patient-oriented evidence } \\
\hline Safety and tolerability & Clear & Nilotinib is well tolerated \\
\hline \multicolumn{3}{|c|}{ Disease-oriented evidence } \\
\hline \multirow[t]{3}{*}{ Cytogenetic response } & Substantial & Nilotinib produces major cytogenetic response in \\
\hline & & $31 \%$ (accelerated phase), $40 \%$ (blastic phase) and \\
\hline & & $\begin{array}{l}57 \% \text { (chronic phase) of patients resistant or intoler- } \\
\text { ant to imatinib. } 100 \% \text { complete response has been } \\
\text { achieved in newly diagnosed patients }\end{array}$ \\
\hline Hematologic response & Substantial & $\begin{array}{l}\text { Nilotinib produces complete hematologic response } \\
\text { in II\% (blastic phase), } 26 \% \text { (accelerated phase), } \\
\text { and } 77 \% \text { (chronic phase) of patients resistant or } \\
\text { intolerant to imatinib }\end{array}$ \\
\hline
\end{tabular}

Correspondence: Elias Jabbour Department of Leukemia, Unit 428, The University of Texas M.D.Anderson Cancer Center, 1515 Holcombe Blvd, Houston, TX 77030, USA Email ejabbour@mdanderson.org 


\section{Scope, aims, and objectives}

Chronic myelogenous leukemia (CML) is characterized by a balanced translocation, involving a fusion of the Abelson oncogene (ABL) from chromosome $9 \mathrm{q} 34$ with the breakpoint cluster region (BCR) on chromosome 22q11.2, $\mathrm{t}(9 ; 22)$ (q34; q11.2), the Philadelphia chromosome (Ph). The molecular consequence of this translocation is the generation of a BCR-ABL fusion oncogene, which in turn translates into a Bcr-Abl oncoprotein. This most frequently has a molecular weight of $210 \mathrm{kD}\left(\mathrm{p} 210^{\mathrm{Bcr} / \mathrm{Abl}}\right)$ and has increased tyrosine kinase activity which is essential to its transforming capability. ${ }^{1,2}$ Imatinib mesylate is a potent and selective tyrosine kinase inhibitor that has become standard therapy for patients with CML in all stages of the disease. ${ }^{2}$ A complete cytogenetic response (CGCR) can be achieved in $50 \%$ to $60 \%$ of patients treated in chronic phase (CP) after failure with interferon alpha (IFN- $\alpha)^{3,4}$ and in over $80 \%$ of those receiving imatinib as first-line therapy. ${ }^{5,6}$ Responses are durable in most patients treated in early $\mathrm{CP}$, particularly among those who achieve major molecular responses (MMR) (eg, $\geq 3$-log reduction in transcript levels). ${ }^{7,8}$

Despite the excellent results with imatinib in CML, resistance to this agent does occur in some cases at an annual rate of approximately $4 \%$ in newly diagnosed CML, but more often in advanced disease. ${ }^{9}$ Resistance may arise in several different ways, including BCR-ABL-dependent and BCR-ABL-independent mechanisms. BCR-ABL kinase domain point mutations are often associated with imatinib resistance. These mutations impair imatinib activity, for example by interfering with an imatinib binding site or by stabilizing a conformation of BCR-ABL with reduced affinity to imatinib. ${ }^{10,11}$ BCR-ABL kinase domain mutations vary in the extent to which they block imatinib binding and induce resistance to this drug. ${ }^{12,13}$

Several approaches have been investigated to overcome resistance to imatinib, including the development of new, more potent tyrosine kinase inhibitors (TKIs).${ }^{14}$ Examples of such inhibitors include nilotinib (Tasigna), ${ }^{15}$ dasatinib, ${ }^{16}$ and other TKIs under clinical investigation such as bosutinib ${ }^{17}$ and INNO-406. ${ }^{18}$ Herein we review the currently available data with nilotinib, including preclinical findings, pharmacokinetic data, results from phase I and II trials, possible indications beyond CML, and potential for use in combination therapy.

\section{Structure}

Nilotinib was developed using a rational design strategy based on the premise that Bcr-Abl inhibitors more potent and selective than imatinib could be developed by making modest changes in this molecule. ${ }^{15}$ Analysis of the structure of imatinib and that of the Abl kinase domain indicated that changes to the structure's part that binds deep into the ATP-binding pocket would be likely to decrease its efficacy, but that modification of the methylpiperazinyl group of imatinib that lies along a partially hydrophobic group on the surface of $\mathrm{Abl}$ kinase might improve binding characteristics. Substitutions in this ring system resulted in the discovery of nilotinib, which is structurally similar to imatinib (Figure 1). ${ }^{19}$

\section{In vitro studies}

Results from in vitro studies have demonstrated that nilotinib is more potent than imatinib in inhibiting Bcr-Abl tyrosine kinase activity in cell lines and that it is at least 10-to 30-fold more potent than imatinib in inhibiting proliferation of Bcr-Abl-expressing cells. Inhibition of cell growth by nilotinib was associated with induction of apoptosis, but it did not decrease the formation of normal human myeloid and erythroid progenitor cells at concentrations $\leq 100 \mathrm{nM}^{15}$

Nilotinib effectively inhibited proliferation of $\mathrm{Ba} / \mathrm{F} 3$ cells stably expressing point mutations (E255V, F317L, M351T, F486S, G250E, M244V, L248R, Q252H, Y253H, E255K, E279K, E282D, V289S, and L384M) associated with imatinib resistance in patients (Figure 2). However, the T315I mutant remained resistant to nilotinib at concentrations $<10 \mu \mathrm{M} .^{15,20}$ Nilotinib also potently inhibited tyrosine autophosphorylation of the E255K, E255V, F317L, M351T, and F486S Bcr-Abl mutants, and these effects were not associated with decreases in Abl or Bcr-Abl protein levels. Overall, these results supported the conclusion that many imatinib-resistant Bcr-Abl mutants were relatively or absolutely more sensitive to nilotinib. ${ }^{21}$

Nilotinib also inhibits to a lesser extent platelet-derived growth factor receptor (PDGFR-alpha) and PDGFR-beta, as well as c-kit dependent cell proliferation. In contrast, imatinib has more potency against PDGFR and c-kit than
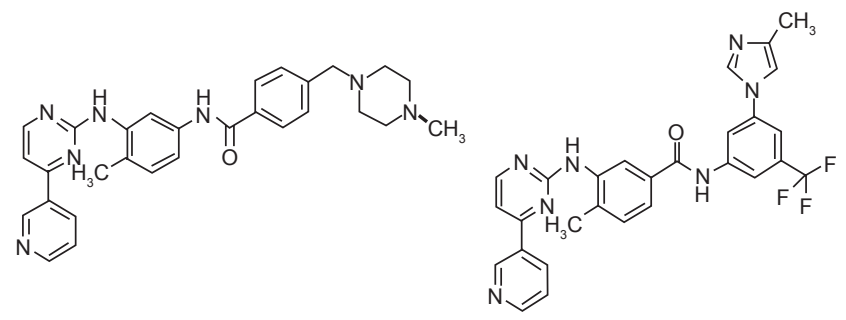

Imatinib

Nilotinib

Figure I Structural formulae of imatinib and nilotinib. ${ }^{19}$ 


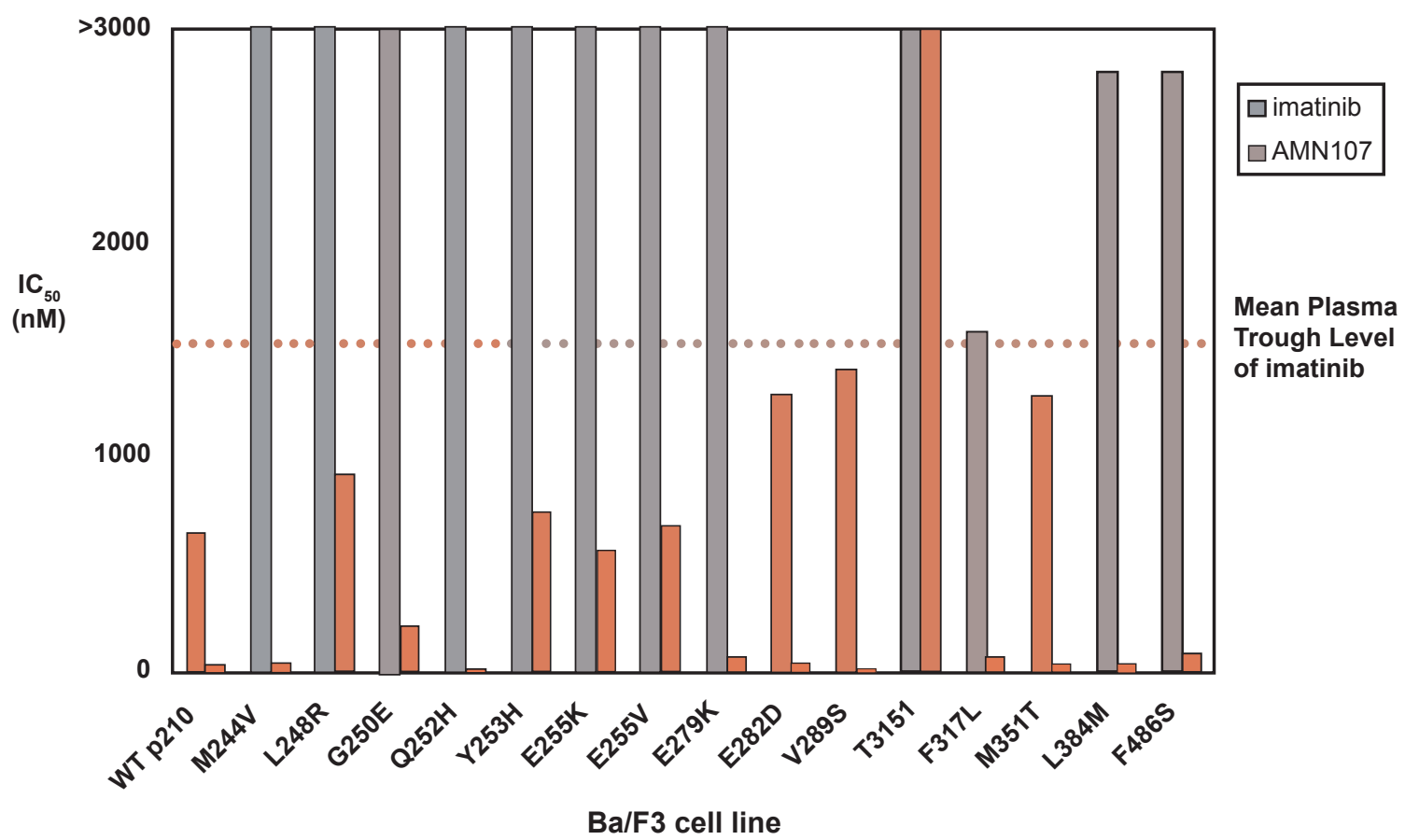

Figure 2 Comparison of imatinib and nilotinib (AMNI07) $I C_{50}$ values for blocking proliferation of Ba/F3 cells expressing non-mutated Bcr-Abl or kinase domain mutated Bcr-Abl. ${ }^{15}$ Copyright $($ C 2005, Elsevier. Reproduced with permission from Weisberg E, Manley PW, Breitenstein W, et al. Characterization of AMNI07, a selective inhibitor of native and mutant Bcr-Abl. Cancer Cell. 2005;7:129-14I.

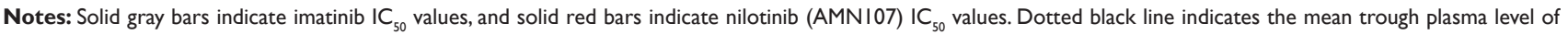
imatinib reported in patients 24 hours following treatment with a once-daily dose of $400 \mathrm{mg}$.

Abl. Nilotinib has no significant activity against other kinases evaluated at concentrations $<3000 \mathrm{nM}^{19}$

Studies investigating the induction of mutants after exposure to imatinib under conditions that favor mutagenesis using a cell-based screen indicated that resistance to nilotinib was associated with a limited spectrum of Bcr-Abl kinase mutations, mostly affecting the P-loop and T315I. With the exception of T315I, all of the mutations identified in one study were effectively suppressed when the nilotinib concentration was increased to $2000 \mathrm{nM}$, which is within the trough-peak range for plasma levels $(1.7-3.6 \mu \mathrm{M})$ measured in patients treated with nilotinib $400 \mathrm{mg}$ twice daily. ${ }^{15,22}$ These results support the view that the clinical use of nilotinib has relatively low potential to result in significant resistance development by Bcr-Abl-expressing cells. ${ }^{22}$ Ray and colleagues have recently reported in a random mutagenesis screen on six mutations (L248V, Q252H, Y253H, Y253C, E255K, and T315I) that can recover after nilotinib incubation; ${ }^{23}$ these mutations have been previously reported in patients receiving imatinib. However, the authors did not recover other clinically identified mutants that confer imatinib resistance (eg, E255V, Y253F, or T315S/G). ${ }^{23}$

Additive/synergistic activity has been reported following the coadministration of imatinib and nilotinib in a panel of wild type and imatinib-resistant Bcr-Abl-expressing cell lines. ${ }^{24}$ This additive activity was confirmed in vivo in mice harboring murine 32D p210 cells. Mice treated with both agents were observed to carry lower tumor burden than mice treated with each agent alone. ${ }^{24}$

\section{In vivo studies}

The efficacy of nilotinib has been documented in in vivo models of CML, such as mice with Bcr-Abl-positive leukemias, both imatinib-sensitive and resistant. In both of these models, treatment with nilotinib significantly decreased tumor burden and prolonged survival relative to vehicle. ${ }^{15}$

\section{Pharmacokinetics}

Results from pharmacokinetic studies in Balb/c mice given single doses of nilotinib 20 or $75 \mathrm{mg} / \mathrm{kg}$ in $10 \% \mathrm{NMP} / 90 \%$ PEG300 by gavage indicated that the drug was orally bioavailable and well absorbed. These studies also demonstrated that nilotinib achieved high concentrations in both the liver and bone marrow. ${ }^{15}$

The pharmacokinetics of nilotinib have been evaluated in a phase I dose-escalation study, in which 119 patients with imatinib-resistant $\mathrm{Ph}+\mathrm{CML}$ or acute lymphoblastic leukemia (ALL) received nilotinib as single oral daily 
doses of $50,100,200,400,800$, or $1200 \mathrm{mg}$, or twice-daily doses of 400 or $600 \mathrm{mg} .{ }^{25}$ The median time to peak serum concentrations of nilotinib was three hours and the mean peak concentration at steady state (achieved at day 8 ) in patients administered $400 \mathrm{mg}$ twice daily was $3.6 \mu \mathrm{M}$. Nilotinib had an apparent half-life of 15 hours. There was a 2-3-fold increase in exposure to nilotinib between the first dose and steady state. Peak concentration and area under the serum concentration-time curve at steady state increased with dose from 50 to $400 \mathrm{mg}$ and reached a plateau with doses greater than $400 \mathrm{mg}$. Nonlinearity with higher nilotinib doses is thought to result from saturation of gastrointestinal absorption; nilotinib $400 \mathrm{mg}$ twice daily resulted in steady-state exposure greater than that observed with a single daily $800 \mathrm{mg}$ dose.$^{25}$ Based on these data, twice daily dosing was selected for phase II studies of nilotinib.

\section{Clinical evidence with nilotinib in CML \\ Efficacy}

The phase I study of nilotinib included 119 patients with imatinib-resistant or intolerant $\mathrm{Ph}+\mathrm{CML}$ or ALL who received nilotinib once daily at doses of 50, 100, 200, 400, 800 , or $1200 \mathrm{mg}$, or 400 or $600 \mathrm{mg}$ twice daily. ${ }^{25}$ Patients in this trial received nilotinib daily unless unacceptable adverse events or disease progression occurred. Intrapatient dose escalation was permitted in patients with an inadequate response and no dose-limiting toxicities (DLTs).

Efficacy results for patients with CML are summarized in Table 1. Overall, 39\% of 33 patients with blastic phase CML achieved a hematologic response (HR), and 27\% a cytogenetic response. Among 46 patients with accelerated phase CML, excluding those with clonal evolution, $72 \%$ achieved a HR, and $48 \%$ had a cytogenetic response, including a major cytogenetic response in $20 \%$. Six of 10 patients with clonal evolution achieved a major cytogenetic response. Of 17 patients with chronic phase CML, $92 \%$ of 12 patients with active disease achieved complete hematologic response (CHR) and cytogenetic response was noted in 53\% (complete in 35\%) (Table 1). ${ }^{26}$

Fifty-one Abl kinase domain mutations were observed in 37 (41\%) of 91 patients who were assessed at baseline. Hematologic and cytogenetic responses were similar in patients with or without mutations and in patients with p-loop or other mutations. The two patients with a T315I mutation did not respond to nilotinib. ${ }^{26}$

The efficacy of nilotinib was confirmed in three ongoing phase II studies in imatinib-resistant or intolerant patients with CML in chronic, accelerated, and blastic phases (Table 2). ${ }^{27-30}$

Three hundred twenty one patients with chronic phase CML (71\% imatinib-resistant; 21\% imatinib-intolerant) treated with nilotinib were evaluable. ${ }^{27}$ Nilotinib was given at the dose of $400 \mathrm{mg}$ twice daily, on an empty stomach, and escalated to $600 \mathrm{mg}$ twice daily for inadequate responses. Complete hematologic response was reported in 158 of 206 patients with active disease at the beginning (77\%). Overall, the major cytogenetic response rate was $57 \% ; 41 \%$ had a complete cytogenetic response. Major cytogenetic responses were observed in 125 (55\%) of the 227 imatinib-resistant patients and in 59 (63\%) of the 94 imatinib-intolerant patients. The median time to complete hematologic response and major cytogenetic response was 1.0 and 2.8 months, respectively. The majority of patients $(84 \%)$ maintained the major cytogenetic response for at least 18 months. The estimated 18-month overall survival rate was $91 \%$.

Table I Phase I hematologic and cytogenetic responses to nilotinib in patients with CML

\begin{tabular}{|c|c|c|c|c|c|c|c|c|c|c|c|}
\hline \multirow[b]{2}{*}{ Results in CML transformation } & \multicolumn{5}{|c|}{ No. (\%) Hematologic response } & \multicolumn{6}{|c|}{ No. (\%) Cytogenetic response } \\
\hline & Total/active $^{a}$ & CHR & MR & RTC & Overall & CR & PR & Minor & Minimal & Major & Overall \\
\hline \multicolumn{12}{|l|}{ Accelerated phase } \\
\hline - Hematologic disease & 46 & 21 & 3 & 9 & $33(72)$ & 6 & 3 & 4 & 9 & $9(20)$ & $22(48)$ \\
\hline - Clonal evolution only ${ }^{\mathrm{b}}$ & $10 / 5$ & 5 & - & - & $5(100)$ & 2 & 4 & I & 2 & $6(60)$ & $9(90)$ \\
\hline Total & $56 / 51$ & 26 & 3 & 9 & $38(74)$ & 8 & 7 & 5 & 11 & $15(27)$ & $31(55)$ \\
\hline \multicolumn{12}{|l|}{ Blastic phase } \\
\hline - Myeloid & 24 & 2 & 2 & 6 & $10(42)$ & 1 & 4 & 2 & - & $(2 I)$ & (29) \\
\hline - Lymphoid & 9 & - & I & 2 & $3(33)$ & I & - & - & I & (II) & $(22)$ \\
\hline Total & 33 & 2 & 3 & 8 & $13(39)$ & 2 & 4 & 2 & I & $6(18)$ & $9(27)$ \\
\hline
\end{tabular}

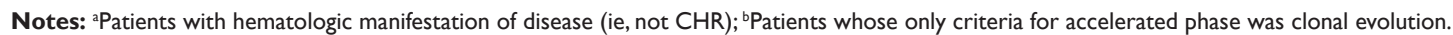
Abbreviations: CHR, complete hematologic response; CR, cytogenetic response; MR molecular response; RTC, return to chronic phase. 
Table 2 Phase II data for nilotinib second-line to imatinib failure

\begin{tabular}{|c|c|c|c|c|c|}
\hline \multirow[b]{3}{*}{ Disease } & \multirow[b]{3}{*}{$\mathbf{n}$} & \multicolumn{3}{|c|}{ \% Response } & \multirow[b]{3}{*}{ Overall survival } \\
\hline & & \multirow[b]{2}{*}{ CHR } & \multicolumn{2}{|c|}{ Cytogenetic response } & \\
\hline & & & Major & Complete & \\
\hline CML Chronic & 321 & 77 & 57 & 41 & $91 \%$ (18 months) \\
\hline CML Accelerated & 136 & 26 & 31 & 19 & $81 \%$ (12 months) \\
\hline CML Blastic & 136 & 11 & 40 & 29 & $42 \%$ ( 12 months) \\
\hline
\end{tabular}

Abbreviations: CHR, complete hematologic response; CML, chronic myeloid leukemia.

In another study, 136 patients with accelerated phase CML received nilotinib at the dose of $400 \mathrm{mg}$ twice daily for a median duration of 210 days. ${ }^{28} \mathrm{~A}$ confirmed hematologic response occurred in 69/129 patients (54\%); 26\% had a complete hematologic response. Major cytogenetic responses occurred in 40/129 patients (31\%); 24/129 patients (19\%) had complete cytogenetic responses. Thirty out of 104 patients (29\%) of imatinib-resistant patients and 10/25 imatinibintolerant patients (40\%), had a major cytogenetic response. Time to first hematologic response and major cytogenetic response was 1 and 2.8 months, respectively. The estimated 12 -month progression free and overall survival rates were $57 \%$ and $81 \%$, respectively.

In the third trial, 136 patients with blastic phase disease (myeloid, $\mathrm{n}=105$; lymphoid, $\mathrm{n}=31$ ) were treated with nilotinib $400 \mathrm{mg}$ twice daily. ${ }^{29}$ The hematologic response rate was $21 \%$ and $11 \%$ achieved complete hematologic response. Major cytogenetic response was achieved in 55 patients (40\%), and 40 (29\%) had complete cytogenetic response. The estimated 12 -month overall survival rate was $42 \%$.

\section{Safety}

In the phase I trial of nilotinib the maximum tolerated dose (MTD), defined as the highest dose given for at least 1 cycle in which $\leq 33 \%$ experienced a DLT, was $600 \mathrm{mg}$ twice daily. ${ }^{24}$ Nilotinib was generally well tolerated. The most common hematologic adverse events across all nilotinib doses were thrombocytopenia (21\%) and neutropenia (14\%), mainly grade 3 or 4 . The frequency of both appeared to increase with nilotinib dose. ${ }^{25}$ Rash and pruritus were the most common nonhematologic adverse events $(20 \%$ and $15 \%$ of patients, respectively), but were almost all grade 1 or $2 .{ }^{25}$ The most common laboratory abnormalities were elevations in bilirubin ( $7 \%$ grade 1 or $2,7 \%$ grade 3 or 4 ), increased lipase ( $5 \%$ grade 3 or 4$)$, and increased aspartate transaminase (AST) and/or alanine transaminase (ALT) (1\% grade 1 or 2 and $3 \%$ grade 3 or 4$).{ }^{25}$ The frequency and grade of bilirubin elevations increased with nilotinib dose, but these rises were generally self-limiting and resolved with continued administration of nilotinib. ${ }^{25}$ Analysis of electrocardiograms (ECGs) indicated one instance of increased QTcF $(5-15 \mathrm{msec})$. One patient had two treatment-related adverse cardiac events (grade 1 pericardial effusion and grade 2 atrial fibrillation). ${ }^{25}$

In all three phase II studies, nilotinib was well tolerated. The rate of grade 3-4 neutropenia was $13 \%$ in patients with chronic phase, $18 \%$ in accelerated phase, and $25 \%$ in blastic phase and $\mathrm{Ph}^{+}$ALL. The corresponding rates of grade 3-4 thrombocytopenia were $13 \%, 27 \%$, and $29 \%$. Nonhematologic side effects were infrequent and usually grade 1-2. These included fatigue, pruritus, headache, muscle spasms, and gastrointestinal disturbances. Rates of grade 3-4 hyperbilirubinemia were $8 \%$, lipase elevation $15 \%$, and hyperglycemia $13 \%$. For these grade 3-4 adverse events, nilotinib was withheld until toxicities recovered to grade 1 or less, and then resumed at a dose of $400 \mathrm{mg}$ daily. Nilotinib was not associated with the common toxic effects seen with imatinib such as fluid retention, edema, cramps, and weight gain, or with pleural effusions. Nilotinib rarely prolonged the QTcF interval. Finally, there was minimal cross intolerance between imatinib and nilotinib. ${ }^{30}$

\section{Frontline therapy}

A recent update of a phase II study in patients with newly diagnosed chronic phase CML showed that nilotinib $400 \mathrm{mg}$ twice daily induces a complete cytogenetic response in nearly all patients as early as 3 months after the start of therapy, with a favorable toxicity profile. Thirty-five patients have been treated for a median of 6.5 months. Complete cytogenetic responses were achieved, respectively, in $96 \%$ and $100 \%$ of patients at 3- and 6-month evaluations. The rate of complete cytogenetic response at 3,6 , and 12 months compares favorably with those observed in historical controls treated with imatinib $400 \mathrm{mg}$ or $800 \mathrm{mg}$ daily: at 12 months $100 \%$ of patients were still in response. Major molecular response was 
observed in $13 \%$ at 3 months, $45 \%$ at 6 months, and $45 \%$ at 12 months. Nilotinib showed generally low and manageable rates of grades $3 / 4$ adverse events. ${ }^{31}$

\section{Combination therapy}

There is growing interest in testing the hypothesis that administration of multiple Abl kinase inhibitors in early-phase patients could be used to delay or prevent the emergence of drug-resistant clones. ${ }^{32}$ The combination of two agents targeting different pathways involved in CML may significantly improve response rates and potentially increase survival.

Support for this concept is provided by preliminary preclinical investigations of the imatinib-nilotinib combination. ${ }^{24}$ Additive/synergistic toxicity against both imatinib-sensitive and imatinib-resistant Bcr-Abl-expressing cells has been reported following coadministration of nilotinib and imatinib, in vitro and in vivo. ${ }^{15,24}$ This cooperative activity could result from pharmacodynamic interactions with cell transporters. Preliminary data suggest that synergy between imatinib and nilotinib may occur at the level of the CML stem cell due to the ability of both imatinib and nilotinib to inhibit or act as substrates of the multidrug efflux transporter Abcg2, which confers resistance toward several anticancer drugs. ${ }^{33}$ It is also reported that imatinib and nilotinib might be taken up in cells by different mechanisms, with the influx, intracellular concentrations of imatinib, and consequently patient sensitivity to imatinib depending upon the organic cation transporter (OCT-1), whereas nilotinib transport appears to be independent of OCT-1. ${ }^{34}$

Both nilotinib and dasatinib efficiently block Bcr-Abl tyrosine kinase catalytic activity by binding to distinct, partially overlapping sites in the kinase domain. Cross resistance with dasatinib is limited to T315I, which is also the only mutant isolated at drug concentrations equivalent to maximal achievable plasma trough levels. ${ }^{20}$ With drug combinations, maximal suppression of resistant clone outgrowth was achieved at lower concentrations compared with single agents, suggesting that such combinations may be equipotent to higher dose single agents. A combination of low doses of dasatinib and low doses of nilotinib may effectively suppress the emergence of mutations other than T315I with an acceptable safety profile..$^{35,36}$ This approach needs to be eventually extended to include specific inhibitors of T315I Bcr-Abl kinase domain mutations. Alternatively, it is also important to explore the potential for synergy between nilotinib and other classes of inhibitors that work through mechanisms not involving inhibition of Abl tyrosine kinase activity. ${ }^{37,38}$
Finally, long-term treatment of CML may require a combination of both conventional and targeted compounds such as tyrosine kinase inhibitors, farnesyl transferase inhibitors, and possibly compounds with other mechanisms of action like vaccines, to stimulate patient immunity and possibly control and eliminate residual disease.

\section{Place of nilotinib in CML therapy}

Imatinib has benefited a large number of patients with CML. However, imatinib resistance has emerged as an important clinical challenge. Novel treatment strategies have been investigated after failure of imatinib therapy. The availability of highly potent tyrosine kinase inhibitors, such as nilotinib, has broadened the treatment armamentarium in CML. Nilotinib appears to overcome imatinib resistance in patients with chronic, accelerated, and blastic phase CML, producing sustained cytogenetic and hematological responses. Combination strategies may be useful, although these have not yet been examined in clinical studies. With the availability of nilotinib, treatment options are increasing in CML, and this is likely to continue in the future.

\section{Disclosure}

The authors report no conflicts of interest in this work.

\section{References}

1. Faderl S, Talpaz M, Estrov Z, O'Brien S, Kurzrock R, Kantarjian HM. The biology of chronic myeloid leukemia. $N$ Engl $J$ Med. 1999;341:164-172.

2. Goldman JM, Melo JV. Chronic myeloid leukemia - advances in biology and new approaches to treatment. $N$ Engl $J$ Med. 2003;349:1451-1464.

3. Kantarjian HM, Cortes JE, O'Brien S, et al. Long-term survival benefit and improved complete cytogenetic and molecular response rates with imatinib mesylate in Philadelphia chromosome-positive chronic-phase chronic myeloid leukemia after failure of interferon-\{alpha\}. Blood. 2004;104:1979-1988

4. Kantarjian H, Sawyers C, Hochhaus A, et al. Hematologic and cytogenetic responses to imatinib mesylate in chronic myelogenous leukemia. $N$ Engl J Med. 2002;346:645-652.

5. O'Brien SG, Guilhot F, Larson RA, et al. Imatinib compared with interferon and low-dose cytarabine for newly diagnosed chronic-phase chronic myeloid leukemia. N Engl J Med. 2003;348: 994-1004.

6. Kantarjian H, Talpaz M, O’Brien S, et al. High-dose imatinib mesylate therapy in newly diagnosed Philadelphia chromosome-positive chronic phase chronic myeloid leukemia. Blood. 2004;103:2873-2878.

7. Cortes J, Talpaz M, O’Brien S, et al. Molecular responses in patients with chronic myelogenous leukemia in chronic phase treated with imatinib mesylate. Clin Cancer Res. 2005;11:3425-3432.

8. Hughes TP, Kaeda J, Branford S, et al. Frequency of major molecular responses to imatinib or interferon alfa plus cytarabine in newly diagnosed chronic myeloid leukemia. $N$ Engl $J$ Med. 2003;349:1423-1432. 
9. Manley PW, Cowan-Jacob SW, Mestan J. Advances in the structural biology, design and clinical development of Bcr-Abl kinase inhibitors for the treatment of chronic myeloid leukaemia. Biochim Biophys Acta. 2005; 1754:3-13.

10. Hochhaus A, La Rosee P. Imatinib therapy in chronic myelogenous leukemia: strategies to avoid and overcome resistance. Leukemia. 2004;18:1-11.

11. Cowan-Jacob SW, Guez V, Griffin JD, et al. Imatinib (STI571) resistance in chronic myelogenous leukemia: molecular basis of the underlying mechanisms and potential strategies for treatment. Mini Rev Med Chem. 2004;4:285-299.

12. Nicolini FE, Corm S, Le QH, et al. Mutation status and clinical outcome of 89 imatinib mesylate-resistant chronic myelogenous leukemia patients: a retrospective analysis from the French intergroup of CML (Fi(varphi)-LMC GROUP). Leukemia. 2006;20:1061-1066.

13. Corbin AS, Buchdunger E, Pascal F, Druker BJ. Analysis of the structural basis of specificity of inhibition of the Abl kinase by STI571. J Biol Chem. 2002;277:32214-32219.

14. Golemovic M, Verstovsek S, Giles F, et al. AMN107, a novel aminopyrimidine inhibitor of Bcr-Abl, has in vitro activity against imatinib-resistant chronic myeloid leukemia. Clin Cancer Res. 2005;11:4941-4947.

15. Weisberg E, Manley PW, Breitenstein W, et al. Characterization of AMN107, a selective inhibitor of native and mutant Bcr-Abl. Cancer Cell. 2005;7:129-141.

16. Talpaz M, Shah N, Kantarjian HM, et al. Dasatinib in imatinibresistant Philadelphia chromosome-positive leukemias. $N$ Engl J Med. 2006;354:2531-2541.

17. Grafone T, Mancini M, Ottaqviani E, et al. A novel 4-anilino3-quinolinecarbonitile dual SRC and ABL kinase inhibitor (SKI-606) has in vitro pro-apoptotic activity on CML Ph blast cells resistant to imatinib. Available at: http://www.parthen-impact. com/cgi-bin/pco/6_05EHA/public/index.cgi?unit+pub_search_ results\&doem_i.

18. Kimura S, Naito H, Segawa H, et al. NS-187, a potent and selective dual $\mathrm{Bcr}-\mathrm{Abl} / \mathrm{Lyn}$ tyrosine kinase inhibitor, is a novel agent for imatinibresistant leukemia. Blood. 2005;106:3948-3954.

19. O’Hare T, Walters DK, Deininger MW, Druker BJ. AMN107: tightening the grip of imatinib. Cancer Cell. 2005;7:117-119.

20. O'Hare T, Walters DK, Stoffregen EP, et al. In vitro activity of Bcr-Abl inhibitors AMN107 and BMS-354825 against clinically relevant imatinib-resistant Abl kinase domain mutants. Cancer Res. 2005;65:4500-4505.

21. Weisberg E, Manley P, Mestan J, Cowan-Jacob S, Ray A, Griffin JD. AMN107 (nilotinib): A novel and selective inhibitor Bcr-Abl. $B r J$ Cancer. 2006;7:129-141.

22. von Bubnoff N, Manley PW, Mestan J, Sanger J, Peschel C, Duyster J. Bcr-Abl resistance screening predicts a limited spectrum of point mutations to be associated with clinical resistance to the Abl kinase inhibitor nilotinib (AMN107). Blood. 2006;108:1328-1333.

23. Ray A, Cowan-Jacob SW, Manley PW, Mestan J, Griffin JD. Identification of BCR-ABL point mutations conferring resistance to the Abl kinase inhibitor AMN107 (nilotinib) by a random mutagenesis study. Blood. 2007;109:5011-5015.
24. Weisberg E, Catley L, Wright RD, et al. Beneficial effects of combining nilotinib and imatinib in preclinical models of BCR-ABL+ leukemias. Blood. 2007;109:2112-2120.

25. Kantarjian H, Giles F, Wunderle L, et al. Nilotinib in imatinib-resistant CML and Philadelphia chromosome-positive ALL. $N$ Engl $\mathrm{J} \mathrm{Med.}$ 2006;354:2542-2551.

26. Kantarjian H, Hochhaus A, Bhalla K, et al. Nilotinib in patients with imatinib-resistant or -intolerant chronic myelogenous leukemia in chronic phase (CML-CP): Updated phase II results [abstract]. J Clin Oncol. 2008;26:7010.

27. le Coutre P, Apperley J, Ottmann OG, et al. Nilotinib in accelerated phase chronic myelogenous leukemia (CML-AP) patients with imatinibresistance or -intolerance: Update of a phase II study [abstract]. $J$ Clin Oncol. 2008;26:7050.

28. Giles F, Kantarjian H, le Coutre P, et al. Nilotinib in patients with Philadelphia chromosome-positive chronic myelogenous leukemia in blast crisis (CML-BC) who are resistant or intolerant to imatinib [abstract]. J Clin Oncol. 2008;26:7017.

29. Ottmann OG, Larson RA, Kantarjian HM, et al. Nilotinib in patients (pts) with relapsed/refractory Philadelphia chromosome-positive acute lymphoblastic leukemia $(\mathrm{Ph}+\mathrm{ALL})$ who are resistant or intolerant to imatinib [abstract]. Blood. 2007;2815.

30. Jabbour E, le Coutre P, Rosti G, et al. Minimal cross-intolerance between nilotinib and imatinib in patients with imatinib-intolerant chronic myelogenous leukemia (CML) in chronic phase (CP) or accelerated phase (AP) [abstract]. J Clin Oncol. 2008;26:7063.

31. Cortes J, Ferrajoli A, Borthakur G, et al. Efficacy of nilotinib (AMN107) in patients (Pts) with newly diagnosed, previously untreated philadelphia chromosome $(\mathrm{Ph})$ - positive chronic myelogenous leukemia in early chronic phase (CML-CP) [abstract]. J Clin Oncol. 2008;26:7016.

32. Shah N, Nicoll J, Branford S, et al. Molecular analysis of dasatinib resistance mechanisms in CML patients identifies novel BCR-ABL mutations predicted to retain sensitivity to imatinib: rationale for combination tyrosine kinase inhibitor therapy [abstract]. Blood. 2005;106:318a.

33. Brendel C, Scharenberg C, Dohse M, et al. Imatinib mesylate and nilotinib (AMN107) exhibit high-affinity interaction with ABCG2 on primitive hematopoietic stem cells. Leukemia. 2007;21:1267-1275.

34. White DL, Saunders VA, Dang P, et al. OCT-1-mediated influx is a key determinant of the intracellular uptake of imatinib but not nilotinib (AMN107): reduced OCT-1 activity is the cause of low in vitro sensitivity to imatinib. i. 2006;108:697-704.

35. Jabbour E, Cortes J, Kantarjian H. Dasatinib for the treatment of Philadelphia chromosome-positive leukaemias. Expert Opin Investig Drugs. 2007;16(5):679-687.

36. Talpaz M, Shah NP, Kantarjian H, et al. Dasatinib in imatinibresistant Philadelphia chromosome-positive leukemias. $N$ Engl J Med. 2006;354:2531-2541

37. O'Hare T, Eide CA, Tyner JW, et al. SGX393 inhibits the CML mutant Bcr-AblT315I and preempts in vitro resistance when combined with nilotinib or dasatinib. Proc Natl Acad Sci U S A. 2008;105:5507-5512.

38. Fiskus W, Pranpat M, Bali P, et al. Combined effects of novel tyrosine kinase inhibitor AMN107 and histone deacetylase inhibitor LBH589 against Bcr-Abl-expressing human leukemia cells. Blood. 2006;108:645-652.
Core Evidence

\section{Publish your work in this journal}

Core Evidence is an international, peer-reviewed open-access journal evaluating the evidence underlying the potential place in therapy of drugs throughout their development lifecycle from preclinical to postlaunch. The focus of each review is to evaluate the case for a new drug or class in outcome terms in specific indications and patient

groups. The manuscript management system is completely online and includes a very quick and fair peer-review system, which is all easy to use. Visit http://www.dovepress.com/testimonials.php to read real quotes from published authors.

\section{Dovepress}

Submit your manuscript here: http://www.dovepress.com/core-evidence-journal 